H. Tasaki

Nagoya Math. J.

Vol. 137 (1995), 33-53

\title{
INTEGRAL GEOMETRY UNDER CUT LOCI IN COMPACT SYMMETRIC SPACES
}

\author{
HIROYUKI TASAKI \\ Dedicated to Professor Masaru Takeuchi on his sixtieth birthday
}

\section{Introduction}

The theory of integral geometry has mainly treated identities between integral invariants of submanifolds in Riemannian homogeneous spaces like as $\int_{G} I(M \cap g N)$ $d \mu_{G}(g)$, where $M$ and $N$ are submanifolds in a Riemannian homogeneous spaces of a Lie group $G$ and $I(M \cap g N)$ is an integral invariant of $M \cap g N$. For example Poincaré's formula is one of typical identities in integral geometry, which is as follows. We denote by $M\left(\mathbf{R}^{2}\right)$ the identity component of the group of isometries of the plane $\mathbf{R}^{2}$ with a suitable invariant measure $\mu_{M\left(\mathbf{R}^{2}\right)}$. The Poincaré's formula for two curves $c_{1}$ and $c_{2}$ in $\mathrm{R}^{2}$ is given by

$$
\int_{M\left(\mathrm{R}^{2}\right)} \#\left(c_{1} \cap g c_{2}\right) d \mu_{M\left(\mathrm{R}^{2}\right)}=2 L\left(c_{1}\right) L\left(c_{2}\right),
$$

where \#(X) denotes the number of the points of $X$ and $L(c)$ denotes the length of c. See I.7.2 Poincaré's formula in [15] for more information about it. Chern [3], Kurita [9], Brothers [2] and Howard [7] extended this formula to the case of Riemann homogeneous spaces. We use the notation in Howard [7]. Let $M$ and $N$ be submanifolds of finite volume in a Riemannian homogeneous space $G / K$ of a Lie group $G$ which satisfy $\operatorname{dim}(G / K) \leq \operatorname{dim} M+\operatorname{dim} N$. Then

$$
\int_{G} \operatorname{vol}(M \cap g N) d \mu_{G}(g)=\int_{M \times N} \sigma_{K}\left(T_{x}^{\perp} M, T_{y}^{\perp} N\right) d \mu_{M \times N}(x, y) .
$$

$\sigma_{K}$ is an integral invariant, which is defined in Section 1 . In the case of $G / K=$ $\mathbf{R}^{2}, \sigma_{K}$ is constant and the above formula implies the Poincaré's one. More generally

Received February 17, 1994 
in the case that $G / K$ is of constant sectional curvature $\sigma_{K}$ is constant and we can get a similar formula as the Poincaré's one. But in general $\sigma_{K}$ is complicated and it is difficult to understand its geometric meanings.

In this paper we shall estimate $\sigma_{K}$ from above instead of exloring its geometric meanings in detail and the volumes of submanifolds from below by the integral of their intersection numbers with the cut loci in a compact simply connected irreducible symmetric space. We shall show the following theorem in Section 3.

THEOREM 3.1. Let $M=G / K$ be a compact simply connected irreducible symmetric space, $p$ be the dimension of a Helgason sphere in $M$ and $N$ be a p-dimensional submanifold of finite volume in $M$. If the condition (1) is satisfied, then we obtain

$$
\int_{G} \#\left(N \cap g C_{o}(M)\right) d \mu_{G}(g) \leq C \operatorname{vol}(N) \operatorname{vol}\left(C_{0}(M)\right) .
$$

The above equality holds if for each point $x$ in $N$ there is a Helgason sphere tangent to $N$ at $x$. If the conditions (1)-(3) are satisfied, then

$$
\operatorname{vol}(S) \leq \frac{1}{C \operatorname{vol}\left(C_{o}(M)\right)} \int_{G} \#\left(N \cap g C_{o}(M)\right) d \mu_{G}(g) \leq \operatorname{vol}(N)
$$

for a Helgason sphere $S$ in $M$ and $N$ whose inclusion map is not null homotopic. In particular, a Helgason sphere is volume minimizing in the class of submanifolds of dimension $p$ whose inclusion maps are not null homotopic.

After Theorem 3.1 we shall apply it to compact symmetric spaces of rank one in Theorem 3.2, compact Hermitian symmetric spaces in Theorem 3.4 and quaternionic Grassmann manifolds in Theorem 5.1. Lê [10] has also applied the method of integral geometry to some estimates of the volume of submanifolds and explicit estimates mainly in Grassmann manifolds.

Concerning Helgason spheres Helgason [5] established these spheres in compact irreducible symmetric spaces. Ohnita [12] proved that Helgason spheres in compact simply connected irreducible symmetric spaces are stable as minimal submanifolds. Lê has proved the volume minimizing property of the Helgason spheres in all compact simply connected irreducible symmetric spaces by a different method, that is, geodesic defect in [11].

I would like to thank Professor Yoshihiro Ohnita for directing my attention to integral geometry and fruitful discussion on the subjects treated in this paper. 


\section{Integral geometry in homogeneous spaces}

In this section we shall mention some definitions and fundamental properties of integral geometry in homogeneous spaces.

Before we mention integral geometry in homogeneous spaces, we give some definitions according to Howard [7]. Let $E$ be a finite-dimensional real vector space with an inner product $\langle$,$\rangle . This inner product naturally induces an inner$ product on the exterior algebra $\wedge^{k} E$ of degree $k$ and its norm on $\wedge^{k} E$ is denoted by $|\cdot|$. If $e_{1}, \ldots, e_{n}$ is an orthonormal basis of $E$, then

$$
e_{i_{1}} \wedge \cdots \wedge e_{i_{k}} \quad\left(1 \leq i_{1}<\cdots<i_{k} \leq n\right)
$$

is an orthonormal basis of $\wedge^{k} E$. For vector subspaces $V$ and $W$ with orthonormal bases $v_{1}, \ldots, v_{p}$ and $w_{1}, \ldots, w_{q}$ respectively, we define $\sigma(V, W)$ by

$$
\sigma(V, W)=\left|v_{1} \wedge \cdots \wedge v_{p} \wedge w_{1} \wedge \cdots \wedge w_{q}\right| .
$$

This is independent of the choice of orthonormal bases.

Lemma 1.1. Let $V$ and $W$ be vector subspaces of a real vector space $E$ with an inner product such that $\operatorname{dim} E=\operatorname{dim} V+\operatorname{dim} W$. Then we obtain

$$
\sigma(V, W)=\sigma\left(V^{\perp}, W^{\perp}\right) .
$$

Proof. Let $\langle$,$\rangle be the inner product and p=\operatorname{dim} V, q=\operatorname{dim} W$. At first we consider the case $V \cap W \neq\{0\}$. In this case $\sigma(V, W)=0$. Since $(V+W)^{\perp} \neq$ $\{0\}$ and $(V+W)^{\perp} \subset V^{\perp} \cap W^{\perp}$, we obtain $\sigma\left(V^{\perp}, W^{\perp}\right)=0$, thus

$$
\sigma(V, W)=\sigma\left(V^{\perp}, W^{\perp}\right) .
$$

Next we consider the case $V \cap W=\{0\}$. In this case there is a linear map $\varphi$ from $W^{\perp}$ to $W$ such that

$$
V=\left\{x+\varphi(x) \mid x \in W^{\perp}\right\} .
$$

Then we obtain

$$
V^{\perp}=\left\{w-{ }^{t} \varphi(w) \mid w \in W\right\} .
$$

Define a symmetric bilinear form $B$ on $W^{\perp}$ by

$$
B(x, y)=\langle\varphi(x), \varphi(y)\rangle \text { for } x, y \in W^{\perp} .
$$

We choose an orthonormal basis $x_{1}, \ldots, x_{p}$ of $W^{\perp}$ which diagonalizes $B$. By this 
choice

$$
\frac{x_{1}+\varphi\left(x_{1}\right)}{\sqrt{1+\left|\varphi\left(x_{1}\right)\right|^{2}}}, \ldots, \frac{x_{p}+\varphi\left(x_{p}\right)}{\sqrt{1+\left|\varphi\left(x_{p}\right)\right|^{2}}}
$$

is an orthonormal basis of $V$ and we obtain

$$
\sigma(V, W)=\prod_{i=1}^{p} \frac{1}{\sqrt{1+\left|\varphi\left(x_{i}\right)\right|^{2}}}
$$

Here

$$
1+\left|\varphi\left(x_{i}\right)\right|^{2}=\left\langle\left(1_{W^{\perp}}+{ }^{t} \varphi \varphi\right)\left(x_{i}\right), x_{i}\right\rangle
$$

and

$$
\prod_{i=1}^{p}\left(1+\left|\varphi\left(x_{i}\right)\right|^{2}\right)=\operatorname{det}\left(1_{W^{\perp}}+{ }^{t} \varphi \varphi\right)
$$

Therefore we obtain

$$
\sigma(V, W)=\operatorname{det}\left(1_{W^{\perp}}+{ }^{t} \varphi \varphi\right)^{-1 / 2}
$$

Similarly

$$
\sigma\left(V^{\perp}, W^{\perp}\right)=\operatorname{det}\left(1_{W}+\varphi^{t} \varphi\right)^{-1 / 2} .
$$

Some calculations on linear algebra imply the identity

$$
\operatorname{det}\left(1_{W^{\perp}}+{ }^{t} \varphi \varphi\right)=\operatorname{det}\left(1_{W}+\varphi^{t} \varphi\right)
$$

and we obtain

$$
\sigma(V, W)=\sigma\left(V^{\perp}, W^{\perp}\right) .
$$

Let $G$ be a Lie group and $K$ be a closed subgroup of $G$. We assume that the image under the linear isotropy representation of $K$ at the origin $o$ of the homogeneous space $G / K$ is compact. Then $G$ has a left invariant Riemannian metric $\langle$,$\rangle which is also invariant under the right multiplication by all elements$ of $K$. Fix such a Riemannian metric $\langle$, $\rangle$ on $G$. It induces a Riemannian metric on $G / K$ which is invariant under the left action of $G$ on $G / K$. Let $X$ be a Riemannian manifold. We denote by $\mu_{X}$ the Riemannian measure induced by the Riemannian metric of $X$. We regard $K$ as a Riemannian manifold with the induced Riemannian metric. For $g, h$ in $G$, a vector subspace $V$ of $T_{g o}(G / K)$ and a vector subspace $W$ of $T_{h o}(G / K)$, we define $\sigma_{K}(V, W)$ by 


$$
\sigma_{K}(V, W)=\int_{K} \sigma\left(d g_{o}^{-1} V, d k_{o}^{-1} d h_{o}^{-1} W\right) d \mu_{K}(k) .
$$

This is independent of the choice of $g$ and $h$. The following theorem is a special case of Poincaré's formula (3.4) in [7].

Theorem 1.2. Let $M$ and $N$ be submanifolds of finite volume in $G / K$. Assume that $\operatorname{dim} M+\operatorname{dim} N=\operatorname{dim}(G / K)$ and that $G$ is unimodular. Then

$$
\int_{G} \#(M \cap g N) d \mu_{G}(g)=\int_{M \times N} \sigma_{K}\left(T_{x}^{\perp} M, T_{y}^{\perp} N\right) d \mu_{M \times N}(x, y),
$$

where the symbol \# $S$ means the number of all elements of a set $S$.

In Section 3 we shall use this formula when $G / K$ is a compact symmetric space and $N$ is the cut locus at the origin of $G / K$.

\section{Cut loci and Helgason spheres}

We shall review the notion of the cut locus of a point in a compact connected Riemannian manifold and some results on the structure of cut loci in a compact symmetric spaces. Using these we shall consider a relation between cut loci and Helgason spheres in a compact symmetric space.

Let $M$ be a compact connected Riemannian manifold and $\operatorname{Exp}_{x}$ denote the exponential map at $x$. For a unit vector $X$ in $T_{x} M, \gamma_{X}(t)=\operatorname{Exp}_{x}(t X)(t \in \mathbf{R})$ is a geodesic parameterized by arc length starting from $x$ with the initial direction $X$. If $t$ is small, $\gamma_{X}$ is a minimizing geodesic joining $x$ and $\gamma_{X}(t)$. If $\left.\gamma_{X}\right|_{\left[0, t_{0}(X)\right]}$ is minimizing and if $\left.\gamma_{X}\right|_{[0, t]}$ is not minimizing for $t>t_{0}(X)$, then $t_{0}(X) X$ is called a tangent cut point of $x$ along $\gamma_{X}$ and $\operatorname{Exp}\left(t_{0}(X) X\right)$ is called a cut point of $x$ along $\gamma_{X}$. The tangent cut locus $\tilde{C}_{x}(M)$ of $x$ is defined by

$$
\tilde{C}_{x}(M)=\bigcup_{\substack{X \in T_{x} M \\|X|=1}} t_{0}(X) X,
$$

which is homeomorphic to a hypersphere in $T_{x} M$. See Kobayashi [8]. The cut locus $C_{x}(M)$ of $x$ is defined by

$$
C_{x}(M)=\operatorname{Exp}_{x}\left(\tilde{C}_{x}(M)\right)
$$

Put

$$
\tilde{B}_{x}(M)=\bigcup_{\substack{X \in T \\|X|=1}}\left\{t X \mid 0 \leq t<t_{0}(X)\right\}
$$


which is homeomorphic to an open ball in $T_{x} M$ and

$$
B_{x}(M)=M-C_{x}(M) .
$$

Then $\operatorname{Exp}_{x}$ induces a diffeomorphism from $\tilde{B}_{x}(M)$ onto $B_{x}(M)$. Therefore $B_{x}(M)$ is homeomorphic to an open ball in $T_{x}(M)$. Thus we obtain a decomposition of $M$ to a disjoint union of an open cell $B_{x}(M)$ and a compact subset $C_{x}(M)$. By this decomposition we obtain the following lemma.

Lemma 2.1. Let $M$ be a compact connected Riemannian manifold and $S$ be a subset of $M$ whose inclusion map is not null homotopic. Then $N \cap C_{x}(M)$ is not empty for any $x$ in $M$.

We shall review the notion of compact symmetric spaces and some results on the structure of their cut loci obtained by Sakai [14] and Takeuchi [16]. Let $G$ be a compact connected Lie group and $\theta$ be an involutive automorphism of $G$. Put

$$
G_{\theta}=\{g \in G \mid \theta(g)=g\} .
$$

For a closed subgroup $K$ of $G$ which lies between $G_{\theta}$ and the identify component of $G_{\theta},(G, K)$ is a compact symmetric pair. Since $G$ is compact, there is a bi-invariant Riemannian metric (, ) on $G$ and it induces a $G$-invariant Riemannian metric on the homogeneous space $M=G / K$, which is also denoted by (, ). Consequently $M$ is a compact symmetric space. Conversely any compact symmetric space is constructed in such a way.

We shall describe the cut locus $C_{o}(M)$ of the origin $o$ in a compact symmetric space $M=G / K$ by a Lie-group theoretical method. Let $g$ and $\mathfrak{t}$ be the Lie algebras of $G$ and $K$ respectively. $\theta$ induces an involutive automorphism of $\mathfrak{g}$, which is also denoted by $\theta$. Then we obtain

$$
\mathfrak{k}=\{X \in g \mid \theta(X)=X\} .
$$

Denote

$$
\mathfrak{p}=\{X \in \mathfrak{g} \mid \theta(X)=-X\} .
$$

We have a direct sum decomposition

$$
g=\mathfrak{l}+\mathfrak{p}
$$

of $\mathfrak{g}$. Take a maximal Abelian subspace $\mathfrak{a}_{\mathfrak{p}}$ in $\mathfrak{p}$ and a maximal Abelian subalgebra $\mathfrak{t}$ in $\mathfrak{g}$ including $\mathfrak{a}_{\mathfrak{p}}$. The complexification $\mathfrak{h}=\mathfrak{t}^{\mathrm{c}}$ of $\mathfrak{t}$ is a Cartan subalgebra of the complexification $\mathfrak{g}^{c}$ of $\mathfrak{g}$. For each element $\alpha$ in the dual space $\mathfrak{h}^{*}$ of $\mathfrak{h}$, put 


$$
\mathfrak{g}_{\alpha}=\{X \in \mathfrak{g} \mid[H, X]=\alpha(H) X \text { for all } H \in \mathfrak{h}\} .
$$

An element $\alpha$ in $\mathfrak{h}^{*}$ is called a root of $\mathfrak{g}^{\mathrm{c}}$ wigh respect to $\mathfrak{h}$ if $\mathfrak{g}_{\alpha} \neq\{0\}$. Let $\Delta$ denote the set of all nonzero zoots of $g^{\mathrm{c}}$. Then we obtain the root space decomposition of $\mathrm{g}^{\mathrm{c}}$ :

$$
\mathfrak{g}^{\mathrm{c}}=\mathfrak{h}+\sum_{\alpha \in \Delta} \mathfrak{g}_{\alpha}
$$

Let $\Delta(\mathfrak{p})$ denote the set of nonzero roots which do not vanish identically on $\mathfrak{a}_{\mathfrak{p}}$. Since $G$ is compact, $\alpha(\mathrm{t}) \subset \sqrt{-1} \mathbf{R}$ for each root $\alpha$ in $\Delta$, so $\alpha$ is real valued on $\sqrt{-1} \mathrm{t}$. We can choose compatible lexicographic orderings on the dual spaces of $\sqrt{-1} \mathrm{t}$ and $\sqrt{-1} a_{\mathfrak{p}}$, which means that a real valued form on $\sqrt{-1} \mathrm{t}$ whose restriction to $\sqrt{-1} a_{\mathfrak{p}}$ is positive is positive. Denote by $\bar{c}$ the restriction of forms on $\mathrm{t}^{\mathrm{c}}$ to $a_{p}^{c}$ and

$$
\Sigma=\{\bar{\alpha} \mid \alpha \in \Delta\}-\{0\}
$$

which is called the root system of the symmetric space $M$ with respect to $\mathfrak{a}_{\mathfrak{p}}$. Now we assume that $M$ is irreducible and simply connected. Then $\Sigma$ is an irreducible root system. Let $\gamma_{0}$ be the highest root in $\Sigma$ and $\left\{\gamma_{1}, \ldots, \gamma_{r}\right\}$ be the fundamental root system of $\sum$, where $r$ is the rank of the symmetric space $M$. Set

$$
\begin{aligned}
& \mathfrak{a}_{s}=\sqrt{-1}\left\{H \in \sqrt{-1} \mathfrak{a}_{\mathfrak{p}} \mid \gamma_{0}(H)=\pi, \gamma_{i}(H) \geq 0 \text { for } 1 \leq i \leq r\right\}, \\
& \mathfrak{a}_{s}^{0}=\sqrt{-1}\left\{H \in \sqrt{-1} \mathfrak{a}_{\mathfrak{p}} \mid \gamma_{0}(H)=\pi, \gamma_{i}(H)>0 \text { for } 1 \leq i \leq r\right\} .
\end{aligned}
$$

Lemma 2.2. Let $M=G / K$ be a compact simply connected irreducible symmetric space. Then the cut locus $C_{o}(M)$ of the origin o in $M$ is described as follows:

$$
C_{o}(M)=\bigcup_{k \in K} k \operatorname{Exp}_{o}\left(a_{s}\right)
$$

The subset $C_{o}^{0}(M)=\cup_{k \in K} k \operatorname{Exp}_{o}\left(a_{s}^{0}\right)$ is a submanifold of $M$ and its complement in $C_{o}(M)$ is stratified by finitely many submanifolds whose dimensions are less than $\operatorname{dim} C_{o}^{0}(M)$.

For the proof of this lemma, see Section 3 of Chapter VII in [6], Theorem 5.3 in [14] or Corollary 3 of Theorem 1.1 in [16]. We can regard the Riemannian measure on $C_{o}^{0}(M)$ of the induced Riemannian metric as a measure on $C_{o}(M)$ with respect to which the measure of $C_{o}(M)-C_{o}^{0}(M)$ is zero.

In order to describe the tangent spaces to $C_{o}^{0}(M)$ we shall define root spaces of the root system $\sum$. For each $\gamma$ in $\sum$, put 
Then we obtain

$$
\tilde{\mathfrak{g}}_{\gamma}=\left\{X \in \mathfrak{g} \mid[H, X]=\gamma(H) X \text { for all } H \in \mathfrak{a}_{\mathfrak{p}}^{c}\right\} .
$$

$$
\tilde{\mathfrak{g}}_{r}=\sum_{\substack{\alpha \in \Delta \\ \alpha=\gamma}} \mathfrak{g}_{\alpha}
$$

Denote by $\Sigma^{+}$the set of all positive roots in $\Sigma$. For each $\gamma$ in $\Sigma^{+}$, put

$$
\mathfrak{p}_{r}=\mathfrak{p} \cap\left(\tilde{\mathfrak{g}}_{r}+\tilde{\mathfrak{g}}_{-r}\right) \text {. }
$$

We get an orthogonal direct sum decomposition of $\mathfrak{p}$ :

$$
\mathfrak{p}=\mathfrak{a}_{\mathfrak{p}}+\sum_{r \in \Sigma^{+}} \mathfrak{p}_{r}
$$

We have the following lemma from the proof of Theorem 3 in [18].

Lemma 2.3. For $k$ in $K$ and $H_{0}$ in $a_{s}^{0}, k \operatorname{Exp}_{o} H_{0}$ is contained in $C_{o}^{0}(M)$ and

$$
T_{k \operatorname{Exp}_{o} H_{0}}\left(C_{o}^{0}(M)\right)=d\left(k \exp H_{0}\right)_{o}\left(\left\{H \in \mathfrak{a}_{\mathfrak{p}} \mid \gamma_{0}(H)=0\right\}+\sum_{\gamma \in \Sigma^{+}-\left\{\gamma_{0}\right\}} \mathfrak{p}_{\gamma}\right) .
$$

We shall define a Helgason sphere and mention some fundamental properties of it. Helgason proved the following theorem in [5].

THEOREM 2.4. Let $M$ be a compact irreducible symmetric space except a real projective space and $\kappa$ be the maximum of the sectional curvatures of $M$. Then there exists a totally geodesic sphere of constant sectional curvature $\kappa$. Any two such sphere of the same dimension are conjugate under the identity component of the group of all isometries of $M$.

Definition 2.5. We call a maximal dimensional sphere mentioned in Theorem 2.4 a Helgason sphere in $M$.

Since the symmetric space $M$ is irreducible, $\mathfrak{g}$ is semisimple, so the Killing form $\langle$,$\rangle of g$ is nondegenerate. For each $\gamma$ in $\sum, A_{r}$, denotes the element in $a_{\mathfrak{p}}^{C}$ satisfying

$$
\left\langle H, A_{\gamma}\right\rangle=\gamma(H) \text { for all } H \in \mathfrak{a}_{\mathfrak{p}}^{\mathrm{c}} \text {. }
$$

We can choose such an $A_{r}$, because $\langle$,$\rangle is nondegenerate on \mathfrak{a}_{\mathrm{p}}^{\mathrm{c}} \cdot \boldsymbol{r}$ is real valued on $\sqrt{-1} a_{\mathfrak{p}}$, so $\sqrt{-1} A_{r}$, is contained in $\mathfrak{a}_{\mathfrak{p}}$. We denote

$$
S=\operatorname{Exp}_{o}\left(\mathbf{R} \sqrt{-1} A_{r_{0}}+\mathfrak{p}_{\gamma_{0}}\right) .
$$


Then $S$ is a Helgason sphere in $M$ and

$$
T_{o}(S)=\mathbf{R} \sqrt{-1} A_{r_{0}}+\mathfrak{p}_{r_{0}} .
$$

By Lemma 2.3 and (2.1) we obtain an orthogonal direct sum decomposition of $T_{o}(M)$ :

$$
T_{o}(M)=T_{o}(S)+d(k \exp H)_{o}^{-1} T_{k \operatorname{ExpH}}\left(C_{o}^{0}(M)\right),
$$

where $k \in K$ and $H \in \mathfrak{a}_{s}^{0}$. In particular,

$$
\operatorname{dim} M=\operatorname{dim} S+\operatorname{dim} C_{o}^{0}(M) .
$$

\section{Integral geometry under cut loci}

We shall show an inequality which estimates the volumes of submanifolds of the same dimension as that of a Helgason sphere from below in a compact simply connected irreducible symmetric space which satisfies certain conditions. Integral geometry under cut loci plays an important role in this estimate.

Let $M=G / K$ be a compact simply connected irreducible symmetric space and $p$ be the dimension of a Helgason sphere in $M$. We use the notation in Section 2. We set

$$
\mathfrak{B}=\mathbf{R} \sqrt{-1} A_{\gamma_{0}}+\mathfrak{p}_{\gamma_{0}}
$$

and denote by $P_{S}$ the orthogonal projection from $\mathfrak{p}$ to $\mathfrak{B}$.

Now we consider the following four conditions concerning the cut locus and Helgason sphere in $\boldsymbol{M}$.

(0) There exists a positive constant $C$ such that

$$
\int_{K}\left|P_{S}(\operatorname{Ad}(k) v)\right|^{p} d \mu_{K}(k)=C|v|^{p} \quad \text { for all } v \in \mathfrak{p}
$$

(1) There exists a positive constant $C$ such that

$$
\int_{K}\left|P_{S}(\operatorname{Ad}(k) v)\right|^{p} d \mu_{K}(k) \leq C|v|^{p} \text { for all } v \in \mathfrak{p}
$$

The equality folds for $v \in \mathfrak{B}$.

(2) For any $k$ in $K$, the map

$$
P_{S} \operatorname{Ad}(k): \mathfrak{B} \rightarrow \mathfrak{B}
$$

is conformal, that is, there is a nonnegative constant $C_{k}$ which satisfies 


$$
\left(P_{S}\left(\operatorname{Ad}(k) u, P_{S} \operatorname{Ad}(k) v\right)=C_{k}(u, v) \text { for all } u, v \in \mathfrak{s}\right. \text {. }
$$

(3) For a Helgason sphere $S$ in $M$,

$$
\#\left(S \cap g C_{o}(M)\right)=1 \text { for almost all } g \in G .
$$

We note that the condition (0) implies (1).

Theorem 3.1. Let $M=G / K$ be a compact simply connected irreducible symmetric space, $p$ be the dimension of a Helgason sphere in $M$ and $N$ be a p-dimensional submanifold of finite volume in $M$. If the condition (1) is satisfied, then we obtain

$$
\int_{G} \#\left(N \cap g C_{o}(M)\right) d \mu_{G}(g) \leq C \operatorname{vol}(N) \operatorname{vol}\left(C_{o}(M)\right) .
$$

The above equality holds if for each point $x$ in $N$ there is a Helgason sphere tangent to $N$ at $x$. If the conditions (1)-(3) are satisfied, then

$$
\operatorname{vol}(S) \leq \frac{1}{C \operatorname{vol}\left(C_{o}(M)\right)} \int_{G} \#\left(N \cap g C_{o}(M)\right) d \mu_{G}(g) \leq \operatorname{vol}(N)
$$

for a Helgason sphere $S$ in $M$ and $N$ whose inchusion map is not mull homotopic. In particular, a Helgason sphere is volume minimizing in the class of submanifolds of dimension $p$ whose inclusion maps are not null homotopic.

Proof. For simplicity we denote $C_{o}$ instead of $C_{o}(M)$ in this proof. Applying Theorem 1.2 to $N$ and $C_{o}$, we obtain

$$
\int_{G} \#\left(N \cap g C_{o}\right) d \mu_{G}(g)=\int_{N \times C_{o}} \sigma_{K}\left(T_{x}^{\perp}(N), T_{y}^{\perp}\left(C_{o}\right)\right) d \mu_{N \times C_{o}}(x, y) .
$$

We shall investigate $\sigma_{K}\left(T_{x}^{\perp}(N), T_{y}^{\perp}\left(C_{o}\right)\right)$ in detail. By the definition (1.1) of $\sigma_{K}$ it is sufficient to consider $\sigma_{K}$ at the origin. For a suitable $g$ in $G$, we obtain $d g_{o}^{-1} T_{y}^{\perp}\left(C_{o}\right)=\mathfrak{g}$ by the orthogonal direct sum decomposition (2.2). Let $V$ be a $p$-dimensional vector subspace of $\mathfrak{p}$. By Lemma $1.1 \sigma\left(V^{\perp}, \mathfrak{g}\right)=\sigma\left(V, \mathfrak{g}^{\perp}\right)$, so $\sigma_{K}\left(V^{\perp}, \mathfrak{s}\right)=\sigma_{K}\left(V, \mathfrak{s}^{\perp}\right)$. We shall estimate the value $\sigma_{K}\left(V, \mathfrak{s}^{\perp}\right)$. Take an orthonormal basis $v_{1}, \ldots, v_{p}$ of $V$. For any $k$ in $K$

$$
\sigma\left(\operatorname{Ad}(k) V, \mathfrak{g}^{\perp}\right)=\left|P_{S} \operatorname{Ad}(k) v_{1} \wedge \cdots \wedge P_{S} \operatorname{Ad}(k) v_{p}\right|,
$$

so we obtain

$$
\sigma_{K}\left(V, \mathfrak{g}^{\perp}\right)=\int_{K}\left|P_{S} \operatorname{Ad}(k) v_{1} \wedge \cdots \wedge P_{S} \operatorname{Ad}(k) v_{p}\right| d \mu_{K}(k)
$$




$$
\begin{aligned}
& \leq \int_{K}\left|P_{S} \operatorname{Ad}(k) v_{1}\right| \cdots\left|P_{S} \operatorname{Ad}(k) v_{p}\right| d \mu_{K}(k) \\
& \leq \prod_{i=1}^{p}\left(\int_{K}\left|P_{S} \operatorname{Ad}(k) v_{1}\right|^{p} d \mu_{K}(k)\right)^{1 / p}
\end{aligned}
$$

by Hölder's inequality. If the condition (1) is satisfied, then the last term is less than or equal to the constant $C$. Therefore

$$
\int_{G} \#\left(N \cap g C_{o}\right) d \mu_{G}(g) \leq C \operatorname{vol}(N) \operatorname{vol}\left(C_{o}\right)
$$

From now on we suppose that the conditions (1)-(3) are satisfied. In the case that $V=\mathfrak{g}$, we obtain

$$
\left|P_{S} \operatorname{Ad}(k) v_{1} \wedge \cdots \wedge P_{S} \operatorname{Ad}(k) v_{p}\right|=\left|P_{S} \operatorname{Ad}(k) v_{1}\right| \cdots\left|P_{S} \operatorname{Ad}(k) v_{p}\right|
$$

for any $k$ in $K$ by (2). Moreover all of the functions $\left|P_{S} \operatorname{Ad}(k) v_{i}\right|$ coincide, so

$$
\int_{K}\left|P_{S} \operatorname{Ad}(k) v_{1}\right| \cdots\left|P_{S} \operatorname{Ad}(k) v_{p}\right| d \mu_{K}(k)=C .
$$

If $S$ is a Helgason sphere in $M$, then we obtain

$$
\int_{G} \#\left(S \cap g C_{o}\right) d \mu_{G}(g)=C \operatorname{vol}(S) \operatorname{vol}\left(C_{o}\right) .
$$

It is equal to $\operatorname{vol}(G)$ by (3), thus

$$
\operatorname{vol}(S)=\frac{\operatorname{vol}(G)}{C \operatorname{vol}\left(C_{o}\right)}
$$

Now we assume that the inclusion map of $N$ to $M$ is not null homotopic. It follows from Lemma 2.1 that $N$ has an intersection with $g C_{o}$ for any $g$ in $G$ and

$$
\operatorname{vol}(G) \leq \int_{G} \#\left(N \cap g C_{o}\right) d \mu_{G}(g) .
$$

Therefore we obtain

$$
\operatorname{vol}(S) \leq \frac{1}{C \operatorname{vol}\left(C_{o}\right)} \int_{G} \#\left(N \cap g C_{o}\right) d \mu_{G}(g) \leq \operatorname{vol}(N) .
$$

Since the inclusion map of $S$ is not null homotopic by [11], $S$ is volume minimizing in the class of submanifolds of dimension $p$ whose inclusion maps are not null homotopic. 
THEOREm 3.2. Let $M$ be one of the complex and quaternionic projective spaces and the Cayley projective plane, $p$ be the dimension of a Helgason sphere in $M$ and $N$ be a $p$-dimensional submanifold of finite volume in $M$. Then we obtain

$$
\int_{G} \#\left(N \cap g C_{o}(M)\right) d \mu_{G}(g) \leq C \operatorname{vol}(N) \operatorname{vol}\left(C_{o}(M)\right) .
$$

The above equality holds if for each point $x$ in $N$ there is a Helgason sphere tangent to $N$ at $x$. Moreover

$$
\operatorname{vol}(S) \leq \frac{1}{C \operatorname{vol}\left(C_{o}(M)\right)} \int_{G} \#\left(N \cap g C_{o}(M)\right) d \mu_{G}(g) \leq \operatorname{vol}(N)
$$

holds for a Helgason sphere $S$ in $M$ and $N$ whose inclusion map is not null homotopic.

Remark. The estimate in Theorem 3.2 for the case of the complex projective spaces is obtained by Lê in Proposition 2.11 of [10]. She also obtained an estimate of the volume of submanifolds of even dimensions in the complex projective spaces in Proposition 3.10 of [10].

Proof. It is known that $M$ is a compact simply connected symmetric space of rank one. A Helgason sphere in $M$ is a projective line in each case. Since the linear isotropy action of $K$ on the unit sphere in $\mathfrak{p}$ is transitive, (0) is satisfied. If $M$ is a complex (quaternionic) projective space, the map $P_{S} \operatorname{Ad}(k): \mathfrak{g} \rightarrow \mathfrak{g}$ is a complex (quaternionic) linear map for any $k$ in $K$, so it satisfies (2). In the case that $M$ is the Cayley projective plane, (2) is proved in the proof of Théorème 6.6 in [1]. The condition (3) follows from projective geometry. A cell decomposition of $M$ implies that the homology class represented by a projective line generates $H_{p}(M ; \mathbf{Z})$, so the inclusion map of a projective line is not null homotopic. Thus we have proved the theorem by Theorem 3.1.

Lemma 3.3. Let $M$ be a compact simply connected irreducible symmetric space whose Helgason sphere is of demension 2. Then (0) is satisfied.

Proof. We define a symmetric bilinear form $B$ on $\mathfrak{p}$ by

$$
B(u, v)=\int_{K}\left(P_{s} \operatorname{Ad}(k) u, P_{S} \operatorname{Ad}(k) v\right) d \mu_{K}(k) \quad \text { for } u, v \in \mathfrak{p} .
$$

The linear isotropy representation of $K$ on $\mathfrak{p}$ is irreducible and $B$ is invariant under this representation. By Schur's lemma there is a positive constant $C$ such 
that

$$
B(u, v)=C(u, v) \quad \text { for } u, v \in \mathfrak{p} .
$$

Therefore

$$
\int_{K}\left|P_{s} \operatorname{Ad}(k) v\right|^{2} d \mu_{K}(k)=C|v|^{2} \text { for } v \in \mathfrak{p} .
$$

which is the condition $(0)$.

THEOREM 3.4. Let $M$ be a compact irreducible Hermitian symmetric space and $N$ be a 2-dimensional submanifold of finite volume in $M$. Then we obtain

$$
\int_{G} \#\left(N \cap g C_{o}(M)\right) d \mu_{G}(g) \leq C \operatorname{vol}(N) \operatorname{vol}\left(C_{o}(M)\right) .
$$

The above equality holds if for each point $p$ in $N$ there is a Helgason sphere tangent to $N$ at $p$. Moreover

$$
\operatorname{vol}(S) \leq \frac{1}{C \operatorname{vol}\left(C_{o}(M)\right)} \int_{G} \#\left(N \cap g C_{o}(M)\right) d \mu_{G}(g) \leq \operatorname{vol}(N)
$$

holds for a Helgason sphere $S$ in $M$ and $N$ whose inclusion map is not null homotopic.

Remark. The estimate in Theorem 3.4 is a generalization of that in Theorem 3.2 for the case of the complex projective spaces.

Proof. It is known that $M$ is simply connected, so we can apply the results obtained above to $M$. By Example 5.11 in [17] a Helgason sphere $S$ in $M$ is a complex submanifold and isometric to a complex projective line. Thus we can apply Lemma 3.3 to $M$ and $(0)$ is satisfied. The map $P_{S} \operatorname{Ad}(k): \mathfrak{g} \rightarrow \mathfrak{g}$ is a complex linear map for any $k$ in $K$, so (2) is satisfied.

A certain irreducible unitary representation of $G$ with representation space $V$ induces a canonical imbedding $M$ into the complex projective space $P(V)$, which consists of all complex lines through 0 in $V$. According to Example 5.11 in [17] the image of $S$ under this imbedding is a complex projective line in $P(V)$, that is, $P(W)$ for a 2-dimensional complex vector subspace $W$ in $V$. By Corollary 8 in [19] $C_{x}(M)=M \cap C_{x}(P(V))$ for $x$ in $M$, so \# $\left(S \cap C_{x}(M)\right)=1$ for $x$ in $M \cap$ $P\left(W^{\perp}\right)$. Therefore

$$
\#\left(S \cap C_{x}(M)\right)=1 \text { for almost all } x \in M,
$$


and (3) is satisfied. Thus we have proved the theorem by Theorem 3.1.

\section{Cut loci in Grassmann manifolds}

We shall show that the condition (3) mentioned in Section 3 holds for the real, complex and quaternionic Grassmann manifolds in this section.

Let $\mathbf{K}$ be one of the fields of complex and quaternionic numbers. The $\mathbf{K}$-Grassmann manifolds $\mathrm{Gr}_{m}\left(\mathbf{K}^{m+n}\right)$ consists of all subspaces of $\mathbf{K}$-dimension $m$ in $\mathbf{K}^{m+n}$. As is well known, $\mathrm{Gr}_{m}\left(\mathbf{K}^{m+n}\right)$ has a Riemannian metric with respect to which it is a compact symmetric space. It is simply connected.

Proposition 4.1. The condition (3) is satisfied for the $\mathbf{K}$-Grassmann manifold $\mathrm{Gr}_{m}\left(\mathbf{K}^{m+n}\right)$.

Proof. Take and fix an orthonormal basis $e_{1}, \ldots, e_{m+n}$ of $\mathbf{K}^{m+n}$

$$
S=\left\{W \in \mathrm{Gr}_{m}\left(\mathbf{K}^{m+n}\right) \mid\left\langle e_{1}, \ldots, e_{m-1}\right\rangle \subset W \subset\left\langle e_{1}, \ldots, e_{m+1}\right\rangle\right\}
$$

is a Helgason sphere of $\mathrm{Gr}_{m}\left(\mathbf{K}^{m+n}\right)$. The cut locus of $V$ in $\mathrm{Gr}_{m}\left(\mathbf{K}^{m+n}\right)$ is given by

$$
C_{V}=\left\{W \in M \mid \operatorname{dim}\left(W \cap V^{\perp}\right) \geq 1\right\} .
$$

See [20] and [21], or [13]. Since

$$
\operatorname{dim}\left\langle e_{1}, \ldots, e_{m+1}\right\rangle=m+1, \operatorname{dim} V^{\perp}=n,
$$

the following inequality holds:

$$
\operatorname{dim}\left(\left\langle e_{1}, \ldots, e_{m+1}\right\rangle \cap V^{\perp}\right) \geq 1 .
$$

We shall investigate the number of the points of $C_{V} \cap S$ separating the cases dependently on $\operatorname{dim}\left(\left\langle e_{1}, \ldots, e_{p-1}\right\rangle \cap V^{\perp}\right)$.

We first assume

$$
\left.\operatorname{dim}\left\langle e_{1}, \ldots, e_{p+1}\right\rangle \cap V^{\perp}\right)=0 .
$$

We denote by $p$ the orthogonal projection to $\left\langle e_{m}, e_{m+1}\right\rangle$ whose domain is restricted to $\left\langle e_{1}, \ldots, e_{m+1}\right\rangle \cap V^{\perp}$.

$$
\begin{aligned}
2 & \geq \operatorname{dim}(\operatorname{Im} p) \\
& =\operatorname{dim}\left(\left\langle e_{1}, \ldots, e_{m+1}\right\rangle \cap V^{\perp}\right)-\operatorname{dim}\left(\left\langle e_{1}, \ldots, e_{m+1}\right\rangle \cap V^{\perp}\right) \\
& =\operatorname{dim}\left(\left\langle e_{1}, \ldots, e_{m+1}\right\rangle \cap V^{\perp}\right)
\end{aligned}
$$

holds by our assumption, so 


$$
1 \leq \operatorname{dim}\left(\left\langle e_{1}, \ldots, e_{m+1}\right\rangle \cap V^{\perp}\right) \leq 2 .
$$

If $\operatorname{dim}\left(\left\langle e_{1}, \ldots, e_{m+1}\right\rangle \cap V^{\perp}\right)=1$ holds, then

$$
C_{V} \cap S=\left\{\left\langle e_{1}, \ldots, e_{m-1}\right\rangle+\left\langle e_{1}, \ldots, e_{m+1}\right\rangle \cap V^{\perp}\right\}
$$

and thus \# $\left(C_{V} \cap S\right)=1$. If $\operatorname{dim}\left(\left\langle e_{1}, \ldots, e_{m+1}\right\rangle \cap V^{\perp}\right)=2$ holds, then for any $W$ in $S$ the dimension of

$$
W \cap\left(\left\langle e_{1}, \ldots, e_{m+1}\right\rangle \cap V^{\perp}\right)=W \cap V^{\perp}
$$

is greater than 0 , so $W$ belongs to $C_{V}$. Thus we get $S \subset C_{V}$.

We next assume

$$
\operatorname{dim}\left(\left\langle e_{1}, \ldots, e_{m-1}\right\rangle \cap V^{\perp}\right) \geq 1 .
$$

For any $W$ in $S, \operatorname{dim}\left(W \cap V^{\perp}\right) \geq 1$ and $W$ belongs to $C_{V}$. Thus we get $S \subset C_{V}$.

By the above argument we get the following equality between two subsets of $\mathrm{Gr}_{m}\left(\mathbf{K}^{m+n}\right)$ :

$$
\begin{aligned}
& \left\{V \mid \#\left(C_{V} \cap S\right)=1\right\} \\
= & \left\{V \mid \operatorname{dim}\left(\left\langle e_{1}, \ldots, e_{m+1}\right\rangle \cap V^{\perp}\right)=0, \operatorname{dim}\left(\left\langle e_{1}, \ldots, e_{m+1}\right\rangle \cap V^{\perp}\right)=1\right\},
\end{aligned}
$$

which is open and dense in $\mathrm{Gr}_{m}\left(\mathbf{K}^{m+n}\right)$. Therefore the condition (3) is satisfied.

\section{Quaternionic Grassmann manifolds}

We shall show that Theorem 3.1 holds for the quaternionic Grassmann man ifolds $M=S p(m+n) / S p(m) \times S p(n)$ in this section. We assume that $m \leq n$. A 4-dimensional sphere $S=S p(2) / S p(1) \times S p(1)$ naturally embedded in $M$ is a Helgason sphere. We set $G=S p(m+n), K=S p(m) \times S p(n)$ in this section.

THEOREM 5.1. Let $M$ be a quaternionic Grassmann manifold and $N$ be a 4-dimensional submanifold of finite volume in $M$. Then we obtain

$$
\int_{G} \#\left(N \cap g C_{o}(M)\right) d \mu_{G}(g) \leq C \operatorname{vol}(N) \operatorname{vol}\left(C_{o}(M)\right) .
$$

The above equality holds if and only if $N$ is a union of some pieces of Helgason spheres in M. Moreover

$$
\operatorname{vol}(S) \leq \frac{1}{C \operatorname{vol}\left(C_{o}(M)\right)} \int_{G} \#\left(N \cap g C_{o}(M)\right) d \mu_{G}(g) \leq \operatorname{vol}(N)
$$


holds for a Helgason sphere $S$ in $M$ and $N$ whose inclusion map is not null homotopic

Proof. We have already proved the condition (3) in the Section 4 . So we shall show the conditions (1) and (2) for the quaternionic symmetric space $M$.

We set

$$
\begin{aligned}
& \mathfrak{k}=\left\{\left[\begin{array}{cc}
A & 0 \\
0 & B
\end{array}\right] \mid A \in S p(m), B \in S p(n)\right\}, \\
& \mathfrak{p}=\left\{\left[\begin{array}{cc}
0 & X \\
-{ }^{t} \bar{X} & 0
\end{array}\right] \mid X \in M(m, n ; \mathbf{H})\right\} .
\end{aligned}
$$

Then $\mathfrak{g}=\mathfrak{k}+\mathfrak{p}$ is a canonical direct sum decomposition for the symmetric space $M$. Since $\mathfrak{p}$ is isomorphic to $M(m, n ; \mathbf{H})$, we identify them. The action of $K$ on $\mathfrak{p}$ through this identification is

$$
\operatorname{Ad}\left[\begin{array}{cc}
A & 0 \\
0 & B
\end{array}\right](X)=A X^{t} \bar{B} \quad(A \in S p(m), B \in S p(n), X \in M(m, n ; \mathbf{H})) .
$$

The tangent space $\mathfrak{B}$ of the Helgason sphere $S$ is

$$
\mathfrak{B}=\left\{\left[\begin{array}{ll}
z & 0 \\
0 & 0
\end{array}\right] \mid z \in \mathbf{H}\right\} \subset \mathfrak{p} .
$$

We identify $\mathfrak{B}$ to the quaternionic numbers $\mathbf{H}$. For $A \in S p(m), B \in S p(n)$ we obtain

$$
P_{S}\left(A\left[\begin{array}{cc}
z & 0 \\
0 & 0
\end{array}\right]{ }^{t} \bar{B}\right)=a_{11} z \bar{b}_{11} .
$$

Therefore the maps $P_{S} \operatorname{Ad}(k): \mathfrak{B} \rightarrow \mathfrak{B}$ for $k \in K$ are the actions of $S p(1) \times$ $S p(1)$ on $\mathbf{H}$ multiplied by real numbers and conformal. Thus we have proved the condition (2). Moreover the determinant of the map $P_{S} \operatorname{Ad}(k): \mathfrak{B} \rightarrow \mathfrak{B}$ is nonnegative, which will be used in the proof of Corollary 5.2.

Now we consider the condition (1). Let

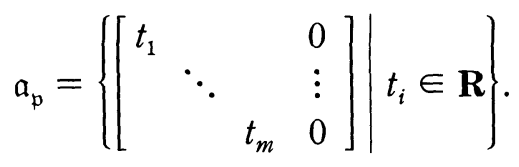

Then $a_{\mathfrak{p}}$ is a maximal Abelian subspace in $\mathfrak{p}$. Since $\int_{K}\left|P_{S} \operatorname{Ad}(k) v\right|^{4} d \mu_{K}(k)$ is invariant under the action of $K$ on $\mathfrak{p}$, it is sufficient to consider its value on $\mathfrak{a}_{\mathfrak{p}}$. 
We take

$$
v=\left[\begin{array}{cccc}
t_{1} & & & 0 \\
& \ddots & & \vdots \\
& & t_{m} & 0
\end{array}\right] \in a_{\mathfrak{p}}
$$

and investigate $\int_{K}\left|P_{S} \operatorname{Ad}(k) v\right|^{4} d \mu_{K}(k)$. Set $k=\left[\begin{array}{cc}A & 0 \\ 0 & B\end{array}\right]$. Since

$$
P_{S}(\operatorname{Ad}(k) v)=P_{S}\left(A\left[\begin{array}{cccc}
t_{1} & & & 0 \\
& \ddots & & \vdots \\
& & t_{m} & 0
\end{array}\right]^{t} \bar{B}\right)=\sum_{i=1}^{m} a_{1 i} t_{i} \bar{b}_{1 i}
$$

we get

$$
\left|P_{S}(\operatorname{Ad}(k) v)\right|^{4}=\sum_{i, j, k, l} t_{i} t_{j} t_{k} t_{l} a_{1 i} \bar{b}_{1 i} b_{1 j} \bar{a}_{1 j} a_{1 l} \bar{b}_{1 l} b_{1 k} \bar{a}_{1 k}
$$

and

$$
\int_{K}\left|P_{S}(\operatorname{Ad}(k) v)\right|^{4} d \mu_{K}(k)=\sum_{i, j, k, l} t_{i} t_{j} t_{k} t_{l} \int_{K} a_{1 i} \bar{b}_{1 i} b_{1 j} \bar{a}_{1 j} a_{1 l} \bar{b}_{1 l} b_{1 k} \bar{a}_{1 k} d \mu_{K}(k) .
$$

This polynomial of $t_{i}$ is invariant under the action of the Weyl group of the symmetric space $M$, it is a linear combination of $\sum_{\imath} t_{i}^{4}$ and $\sum_{i<j} t_{i}^{2} t_{j}^{2}$. Its coefficient of $t_{1}^{4}$ is

$$
\int_{K}\left|a_{11}\right|^{4}\left|b_{11}\right|^{4} d \mu_{K}(k)
$$

The integrand of its coefficient of $t_{1}^{2} t_{2}^{2}$ is

$$
4\left|a_{11}\right|^{2}\left|b_{11}\right|^{2}\left|a_{12}\right|^{2}\left|b_{12}\right|^{2}+2 \Re\left(\left(a_{11} \bar{b}_{11} b_{12} \bar{a}_{12}\right)^{2}\right),
$$

where $\Re z$ is the real part of $z$. The equation $\Re\left(z^{2}\right)=2(\Re z)^{2}-|z|^{2}$ holds for $z \in$ $\mathbf{H}$, the above integrand is

$$
2\left|a_{11}\right|^{2}\left|b_{11}\right|^{2}\left|a_{12}\right|^{2}\left|b_{12}\right|^{2}+4\left(\Re\left(a_{11} \bar{b}_{11} b_{12} \bar{a}_{12}\right)\right)^{2} .
$$

We have $\Re\left(a_{11} \bar{b}_{11} b_{12} \bar{a}_{12}\right)=\Re\left(\left(\bar{a}_{12} a_{11} \bar{b}_{11} b_{12}\right)\right.$. We can change the variables to

$$
\left[\begin{array}{ll}
a_{11} & a_{12} \\
a_{21} & a_{22}
\end{array}\right]\left[\begin{array}{ll}
1 & 0 \\
0 & \mathbf{i}
\end{array}\right]=\left[\begin{array}{ll}
a_{11} & a_{12} \mathbf{i} \\
a_{21} & a_{22} \mathbf{i}
\end{array}\right],
$$

because the measure we use is invariant. We can also do that for $\mathbf{j}, \mathbf{k}$ and get 


$$
\begin{aligned}
& \int_{K} 4\left(\Re\left(\left(a_{11} \bar{b}_{11} b_{12} \bar{a}_{12}\right)\right)^{2} d \mu_{K}(k)\right. \\
= & \int_{K}\left(\left(\Re\left(\left(\bar{a}_{12} a_{11} \bar{b}_{11} b_{12}\right)\right)^{2}+\left(\Re\left((-\mathbf{i}) \bar{a}_{12} a_{11} \bar{b}_{11} b_{12}\right)\right)^{2}\right.\right. \\
& \left.\quad+\left(\Re\left((-\mathbf{j}) \bar{a}_{12} a_{11} \bar{b}_{11} b_{12}\right)\right)^{2}+\left(\Re\left((-\mathbf{k}) \bar{a}_{12} a_{11} \bar{b}_{11} b_{12}\right)\right)^{2}\right) d \mu_{K}(k) \\
= & \int_{K}\left|\bar{a}_{12} a_{11} \bar{b}_{11} b_{12}\right|^{2} d \mu_{K}(k) \\
= & \int_{K}\left|a_{12}\right|^{2}\left|a_{11}\right|^{2}\left|b_{11}\right|^{2}\left|b_{12}\right|^{2} d \mu_{K}(k) .
\end{aligned}
$$

Therefore the coefficient of $t_{1}^{2} t_{2}^{2}$ is

$$
3 \int_{K}\left|a_{11}\right|^{2}\left|a_{12}\right|^{2}\left|b_{11}\right|^{2}\left|b_{12}\right|^{2} d \mu_{K}(k)=3 \int_{S p(m)}\left|a_{11}\right|^{2}\left|a_{12}\right|^{2} \int_{S p(n)}\left|b_{11}\right|^{2}\left|b_{12}\right|^{2} .
$$

We change the variables to

$$
\left[\begin{array}{ll}
a_{11} & a_{12} \\
a_{21} & a_{22}
\end{array}\right]\left[\begin{array}{cc}
1 / \sqrt{2} & 1 / \sqrt{2} \\
1 / \sqrt{2} & -1 / \sqrt{2}
\end{array}\right]=\frac{1}{\sqrt{2}}\left[\begin{array}{ll}
a_{11}+a_{12} & a_{11}-a_{12} \\
a_{21}+a_{22} & a_{21}-a_{22}
\end{array}\right]
$$

and get

$$
\int_{S p(m)}\left|a_{11}\right|^{2}\left|a_{12}\right|^{2}=\frac{1}{4} \int_{S p(m)}\left(\left|a_{11}\right|^{4}+2\left|a_{11}\right|^{2}\left|a_{12}\right|^{2}+\left|a_{12}\right|^{4}-4\left(\Re\left(a_{11} \bar{a}_{12}\right)\right)^{2}\right) .
$$

Thus we obtain

$$
\int_{S p(m)}\left|a_{11}\right|^{2}\left|a_{12}\right|^{2}=\frac{1}{2} \int_{S p(m)}\left(\left|a_{11}\right|^{4}+\left|a_{12}\right|^{4}-4\left(\Re\left(a_{11} \bar{a}_{12}\right)\right)^{2}\right) .
$$

Using (5.1) and get

$$
\int_{S p(m)} 4\left(\Re\left(a_{11} \bar{a}_{12}\right)\right)^{2}=\int_{S p(m)}\left|a_{11}\right|^{2}\left|a_{12}\right|^{2}
$$

and

$$
\int_{S p(m)}\left|a_{11}\right|^{2}\left|a_{12}\right|^{2}=\frac{1}{3} \int_{S p(m)}\left(\left|a_{11}\right|^{4}+\left|a_{12}\right|^{4}\right) .
$$

By the change of the variables:

$$
\left[\begin{array}{ll}
a_{11} & a_{12} \\
a_{21} & a_{22}
\end{array}\right]\left[\begin{array}{ll}
0 & 1 \\
1 & 0
\end{array}\right]=\left[\begin{array}{ll}
a_{12} & a_{11} \\
a_{22} & a_{21}
\end{array}\right]
$$


we get

$$
\int_{S p(m)}\left|a_{11}\right|^{4}=\int_{S p(m)}\left|a_{12}\right|^{4}
$$

and thus

$$
\int_{S p(m)}\left|a_{11}\right|^{2}\left|a_{12}\right|^{2}=\frac{2}{3} \int_{S p(m)}\left|a_{11}\right|^{4}
$$

Similarly

$$
\int_{S p(n)}\left|b_{11}\right|^{2}\left|b_{12}\right|^{2}=\frac{2}{3} \int_{S p(n)}\left|b_{11}\right|^{4}
$$

Thus we have got that the coefficient of $t_{1}^{2} t_{2}^{2}$ is

$$
\frac{4}{3} \int_{K}\left|a_{11}\right|^{4}\left|b_{11}\right|^{4} d \mu_{K}(k) .
$$

By the coefficients obtained above we get

$$
\begin{aligned}
\int_{K}\left|P_{S}(\operatorname{Ad}(k) v)\right|^{4} d \mu_{K}(k) & =\int_{K}\left|a_{11}\right|^{4}\left|b_{11}\right|^{4} d \mu_{K}(k)\left(\sum_{i} t_{i}^{4}+\frac{4}{3} \sum_{i<j} t_{i}^{2} t_{j}^{2}\right) \\
& \leq \int_{K}\left|a_{11}\right|^{4}\left|b_{11}\right|^{4} d \mu_{K}(k)\left(\sum_{i} t_{i}^{2}\right)^{2} \\
& =\int_{K}\left|a_{11}\right|^{4}\left|b_{11}\right|^{4} d \mu_{K}(k)|v|^{4} .
\end{aligned}
$$

Set

$$
C=\int_{K}\left|a_{11}\right|^{4}\left|b_{11}\right|^{4} d \mu_{K}(k) .
$$

Then we get

$$
\int_{K}\left|P_{S}(\operatorname{Ad}(k) v)\right|^{4} d \mu_{K}(k) \leq C|v|^{4} .
$$

The above equality holds if and only if all $t_{i}$ except one are 0 , which means that $v$ belong to $\operatorname{Ad}(k) \mathfrak{g}$ for some $k$ in $K$. Thus we have proved the condition (1).

In the estimate of $\int_{K}\left|P_{S} \operatorname{Ad}(k) v_{1} \wedge \cdots \wedge P_{S} \operatorname{Ad}(k) v_{p}\right| d \mu_{K}(k)$ mentioned in the proof of Theorem 3.1 for $p=4$, if all of $v_{i}$ do not belong to a same $\operatorname{Ad}(k) \mathfrak{g}$ for some $k$ in $K$, the inequality 


$$
\int_{K}\left|P_{S} \operatorname{Ad}(k) v_{1} \wedge \cdots \wedge P_{S} \operatorname{Ad}(k) v_{p}\right| d \mu_{K}(k)<C
$$

holds. Therefore the equality

$$
\int_{G} \#\left(N \cap g C_{o}\right) d \mu_{G}(g)=C \operatorname{vol}(N) \operatorname{vol}\left(C_{o}\right)
$$

holds if and only if for each point $x$ in $N$ there is a Helgason sphere tangent to $N$ at $x$. By Proposition 5.1 in Ohnita [12] the above equality holds if and only if $N$ is a union of some pieces of Helgason spheres in $M$.

COROLLARY 5.2. We choose and fix an orientation of the Helgason sphere $S$ in the quaternionic Grassmann manifold $M$. We denote by $\overrightarrow{\mathfrak{B}}$ the wedge product of a positively ordered orthonormal basis of $\mathfrak{B}$ and define a 4-form $\omega$ on $\mathfrak{p}$ by

$$
\omega\left(X_{1}, X_{2}, X_{3}, X_{4}\right)=\frac{1}{C} \int_{K}\left\langle\operatorname{Ad}(k)\left(X_{1} \wedge X_{2} \wedge X_{3} \wedge X_{4}\right), \overrightarrow{\mathfrak{g}}\right\rangle d \mu_{K}(k)
$$

for $X_{i} \in \mathfrak{p}$. Then $\omega$ can be extended to a parallel 4-form on $M$, denoted the same symbol $\omega$, and $\omega$ is a calibration. $\omega$ calibrates the Helgason sphere and $* \omega$ calibrates the cut locus in M. Both of the Helgason sphere and the cut locus are volume minimizing in their homology classes of real coefficient.

Proof. By the definition of $\omega$, it is invariant under the action of $K$. So we can extend $\omega$ to a parallel form on $M$, because $M$ is a symmetric space. In particular the extended $\omega$ is closed. Let $X_{1}, X_{2}, X_{3}, X_{4}$ be orthonormal vectors in $\mathfrak{p}$.

$$
\begin{aligned}
\omega\left(X_{1}, X_{2}, X_{3}, X_{4}\right) & \leq \frac{1}{C} \mid \int_{K}\left\langle\operatorname{Ad}(k)\left(X_{1} \wedge X_{2} \wedge X_{3} \wedge X_{4}\right), \overrightarrow{\mathfrak{s}}\right\rangle d \mu_{K}(k) \\
& \leq \frac{1}{C} \int_{K}\left|\left\langle\operatorname{Ad}(k)\left(X_{1} \wedge X_{2} \wedge X_{3} \wedge X_{4}\right), \overrightarrow{\mathfrak{s}}\right\rangle\right| d \mu_{K}(k) \\
& \leq \frac{1}{C} \int_{K}\left|P_{S} \operatorname{Ad}(k)\left(X_{1} \wedge X_{2} \wedge X_{3} \wedge X_{4}\right)\right| d \mu_{K}(k)
\end{aligned}
$$

which is already estimated in the proof of Theorem 5.1. Combining this with the fact that the determinant of the $\operatorname{map} P_{S} \operatorname{Ad}(k): \mathfrak{B} \rightarrow \mathfrak{g}$ is nonnegative, which is also mentioned in the proof of Theorem 5.1, we can see that $\omega$ is a calibration and calibrates the Helgason sphere $S$. By (2.2) * $\omega$ calibrates the cut locus in $M$. The volume minimizing property of the Helgason sphere and the cut locus is a consequence of a general theory of calibrations. See Harvey-Laswon [4]. 


\section{REFERENCES}

[1] M. Berger, Du côté de chez Pu, Ann. scient. Éc. Norm. Sup., 5 (1972), 1-44.

[2] J. E. Brothers, Integral geometry in homogeneous spaces, Trans. A. M. S., 124 (1966), 480-517.

[3] S. S. Chern, On integral geometry in Klein spaces, Ann. Math., 43 (1942), $178-189$.

[4] R. Harvey and H. B. Lawson, Jr., Calibrated geometries, Acta Math., 148 (1982), $47-157$.

[5] S. Helgason, Totally geodesic spheres in compact symmetric spaces, Math. Ann., 165 (1966), 309-317.

[6] - Differential geometry, Lie groups, and symmetric spaces, Academic Press, New York, 1978.

[7] R. Howard, Classical integral geometry in Riemannian homogeneous spaces, Contemporary Math., 63 (1987), 179-204.

[8] S. Kobayashi, On conjugate and cut loci, in Studies in global geometry and analysis, (S. S. Chern, ed.), MAA 1967, 96-122.

[9] M. Kurita, An extension of Poincaré formula in integral geometry, Nagoya Math. J., 2 (1950), 55-61.

[10] Lê Hông Vân, Application of integral geometry to minimal surfaces, Int. J. Math., 4 (1993), 89-111.

[11] - Curvature estimate for the volume growth of globally minimal submanifolds, Math. Ann., 296 (1993), 103-118.

[12] Y. Ohnita, On stability of minimal submanifolds in compact symmetric spaces, Compositio Math., 64 (1987), 157-189.

[13] T. Sakai, On the cut loci of compact symmetric spaces, Hokkaido Math. J., 6 (1977), $136-161$.

[14] - On the structure of cut loci in compact Riemannian symmetric spaces, Math. Ann., 235 (1978), 129-148.

[15] L. A. Santaló, Integral geometry and geometric probability, Addison-Wesley, London, 1976.

[16] M. Takeuchi, On conjugate loci and cut loci of compact symmetric spaces I, Tsukuba J. Math., 2 (1978), 35-68.

[17] - Basic transformations of symmetric $R$-spaces, Osaka J. Math., 25 (1988), 259-297.

[18] H. Tasaki, Certain minimal or homologically volume minimizing submanifolds in compact symmetric spaces, Tsukuba J. Math., 9 (1985), 117-131.

[19] - The cut locus and the diastasis of a Hermitian symmetric space of compact type, Osaka J. Math., 22 (1985), 863-870.

[20] Y. C. Wong, Differential geometry of Grassmann manifolds, Proc. Nat. Acad. Sci. U. S. A., 57 (1967), 589-594.

[21] - Conjugate loci in Grassmann manifolds, Bull. A. M. S., 74 (1968), 240-245.

Institute of Mathematics

University of Tsukuba

Tsukuba, Ibaraki

305 Japan

e-mail: tasaki@math.tsukuba.ac.jp 